\title{
Investigation of the kynurenine pathway in Indoleamine 2, 3 dioxygenase deficient mice with inflammatory arthritis
}

\author{
Lukasz Kolodziej
}

Received: 5 August 2012/Accepted: 4 February 2013/Published online: 17 February 2013

(C) The Author(s) 2013. This article is published with open access at Springerlink.com

\begin{abstract}
Tryptophan is an essential amino acid involved in the protein synthesis, cognition, and immunity. Oxidative catabolism of tryptophan is executed by the sets of biochemical reactions collectively referred to as the kynurenine pathway. In the immune system, two distinct enzymes, Indoleamne 2,3 dioxygenase 1 (IDO1) and Indoleamine 2, 3 dioxygenase 2 (IDO2) can initiate metabolic flux through the kynurenine pathway. Rheumatoid arthritis is an autoimmune disease driven by the exacerbated immune response towards self antigens and characterized by the chronic inflammatory reaction of the diarthrodial joints. Collagen induced arthritis (CIA) is an animal model of rheumatoid arthritis. Using CIA in wild type (WT) and mice deficient with Indoleamine 2,3 dioxygenase (Ido1KO), it was of interest to test the impact of Ido1 deletion on the concentration of tryptophan and its
\end{abstract}

Electronic supplementary material The online version of this article (doi:10.1007/s11248-013-9696-5) contains supplementary material, which is available to authorized users.

\section{Kolodziej}

The Kennedy Institute of Rheumatology Division, Imperial College London, Arthritis Research UK Building, Charing Cross Hospital Campus, 65 Aspenlea Road, London W6 8LH, UK

\section{Kolodziej ( $\square$ )}

Department of Clinical Immunology Polish-American, Institute of Pediatrics, Jagiellonian University Medical College, Wielicka Steet 265, 30-663 Kraków, Poland e-mail: lukasz.kl@interia.pl catabolites as well as on mRNA expression for other genes on the kynurenine pathway. Here, when compared with samples taken from naïve WT animals and those with CIA, it was found that only in the inguinal lymph nodes (iLN) taken from Ido1KO mice with CIA tryptophan concentration was significantly increased. In contrast, mRNA expression for Ido2 was decreased in naïve as well as in the diseased iLN taken from Ido1KO mice. Deletion of Idol and reduced mRNA expression for Ido2 neither affected the concentration of the downstream metabolites of tryptophan nor mRNA expression for downstream genes on the kynurenine pathway in iLN. Moreover, the concentration of kynurenine in sera of mice with CIA was significantly decreased in Ido1KO mice with arthritis.

Keywords IDO1 - IDO2 - Transgenic mice · Immunity

\section{Introduction}

Tryptophan is an essential amino acid implicated in the regulation of various biological processes including mood (Russo et al. 2003) and immunity (Moffett and Namboodiri 2003). Indoleamnine 2, 3 dioxygenase 1 (IDO1) is an initial enzyme which catalyses oxidative degradation of tryptophan via the kynurenine pathway (Fig. 1) (Hayaishi 1976). Hence, not surprisingly, IDO1 and the kynurenine pathway have been extensively investigated (Kolodziej et al. 2011). Therefore, mice 
deficient in Idol (Ido1KO) are a vital tool in the research on IDO1 mediated tryptophan degradation (Baban et al. 2004). Moreover, another enzyme, Indoleamine 2, 3 dioxygenase 2 (IDO2), which is also able to catalyze oxidative catabolism of tryptophan via the kynurenine pathway, has been recently discovered (Metz et al. 2007). Interestingly, IdoI and Ido2 are located on the same chromosome in the close proximity to each other (Ball et al. 2007). Hence, it could be predicted deletion of Idol could be compensated by the increased expression of Ido 2 mRNA, resulting in the decreased concentration of tryptophan in Ido1KO mice despite inactivation of IDO1.

In the immune system, tryptophan has been implicated in the immune regulation (Moffett and Namboodiri 2003). The decreased concentration of this amino acids leads to cell cycle arrest (Frumento et al. 2002; Munn et al. 1999) and apoptosis (Fallarino et al. 2002). In addition, toxic byproducts of tryptophan degradation via the kynrenine pathway are potent inducers of apoptosis in T cells (Kolodziej et al. 2011; Terness et al. 2002). IDO1 mediated tryptophan catabolism has been also proven to be important in the biology of Th17 and Treg cells (Baban et al. 2009; Sharma et al. 2009). Th17 cells exacerbate inflammation (Weaver et al. 2006), whereas

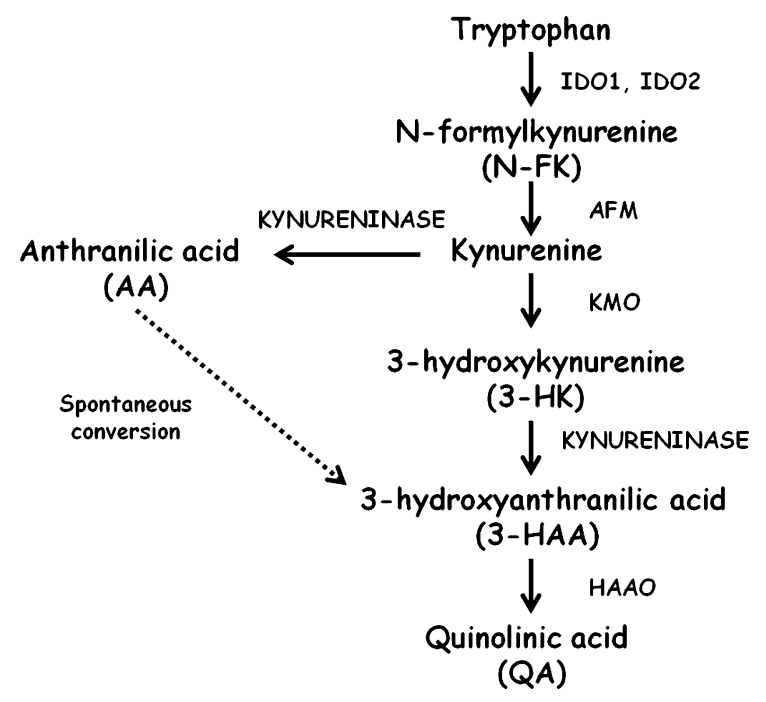

Fig. 1 Simplified schematic representation of the kynurenine pathway. Enzymes involved in tryptophan metabolism via the kynurenine pathway: indoleamine 2,3 dioxygenase 1 (EC 1.13.11.17) IDO1, indoleamine 2,3 dioxygenase 2 (EC 1.13.11.52) IDO2, arylformamidase (EC 3.5.1.9) AFM, kynurenine 3-monooxygenase (EC 2.6.1.7) KMO, Kynureninase (EC 3.7.1.3), 3-hydroxyanthranilate 3,4 dioxygenase (EC 1.13.11.6) HAAO. Spontaneous conversion of AA into 3-HAA has been previously reported (Ueda et al. 1978)
Treg cells exhibit potent anti-inflammatory activity (Zaiss et al. 2007). Interestingly, tryptophan starvation accompanied with accumulation of its toxic byproducts e.g. anthranilic acid (AA) and 3-hydroxyanthranilic acid (3-HAA) has been shown to abrogate function of Th17 cells in a dose dependent manner (Desvignes and Ernst 2009). In addition, the same conditions have been proven to promote the development of Treg's (Fallarino et al. 2006). Thus, in the context of metabolic regulation, Ido1KO mice may be also a useful tool in the investigations focused on the reciprocal functional relations between Th17 and Treg cells.

Rheumatoid arthritis (RA) is an autoimmune systemic disease affecting around $1 \%$ of the western population (Smolen and Aletaha 2009). In RA, activity of Th17 cells takes over an anti-inflammatory role of Treg cells and chronic inflammation of the joints progress (Sato et al. 2006). Collagen induced arthritis (CIA) is an animal model of RA (Inglis et al. 2007) driven by the execrated function of Th17 cells and abnormally low activity of Treg (Park et al. 2011). In Ido1KO mice with CIA the incidence of the disease has been found to be higher than in the wild type (WT) diseased mice (Criado et al. 2009). In addition, the severity of symptoms was higher in Ido1KO mice with CIA than in the WT animals with CIA (Criado et al. 2009). Thus, taken together, it was of interest to test if deletion of Idol could be: (1) compensated by the increased mRNA expression for Ido2 in iLN, (2) impact mRNA expression for downstream genes on kynurenine pathway (Afm, Kmo, Kynu, and Haao) in iLN during CIA, (3) influence the concentration of tryptophan and its anti inflammatory catabolites: kynurenine, AA, and 3-HAA in iLN from Ido1KO mice with CIA, and (4) coincide with reduced concentration of kynurenine in serum of Ido1KO mice with CIA.

\section{Materials and methods}

Animals, CIA development and tissue harvesting

All experimental procedures were approved by the UK Home Office. Adult C57BL/6J mice (aged 10-12 weeks) were used in experiments (Charles River, UK). CIA was induced as previously described (Inglis et al. 2007). Ido1KO mice were kept and bred in the Biological Service Unit in the Kennedy Institute of Rheumatology. Mice were humanely sacrificed and lymph nodes were immediately frozen and kept at $-80{ }^{\circ} \mathrm{C}$. 
HPLC analysis and kynurenine measurements

Concentration of tryptophan, AA, and 3-HAA was determined with HPLC method. The HPLC system (UltiMate 3000) was provided by Dionex, UK. All chromatographic procedures were performed in $37^{\circ} \mathrm{C}$, with C18 column (Acclaim 120, Dionex, UK) $3 \mu \mathrm{m}$, $120 \AA$; $4.6 \times 150 \mathrm{~mm}$, and injection volume of $10 \mu \mathrm{L}$. Tryptophan concentration was determined by HPLC with fluorescence detection (excitation $\lambda=284 \mathrm{~nm}$; emission $\lambda=365 \mathrm{~nm})$. The mobile phase $(1 \mathrm{ml} / \mathrm{min}$ flow rate) consisted of $50 \mathrm{mM}$ acetic acid, $100 \mathrm{mM}$ zinc acetate, and $3 \%$ acetonitrile). Concentartion of AA and 3-HAA was determined by HPLC with fluorescence detection (excitation $\lambda=320 \mathrm{~nm}$; emis$\operatorname{sion} \lambda=420 \mathrm{~nm}$ ). The mobile phase $(1 \mathrm{ml} / \mathrm{min}$ of flow rate) consisted of $25 \mathrm{mM}$ sodium acetate (Sigma), $1 \mathrm{mM}$ acetic acid (pH5.5). Kynurenine concentration was assessed by a colorimetric assay (Hara et al. 2008).

RNA isolation, preparation of cDNA, and qRTPCR

RNA was extracted using RNA-Stat60 reagent (AMS Biotechnology) according to the manufacturer's instructions. cDNA was transcribed using the Applied Biosystems Reverse Transcription System. The total volume of qRT-PCR reaction was $10 \mu \mathrm{l}$. TaqMan primer probes were provided by Applied Biosystems UK. List of primers is shown in the supplementary table 1. PCR reaction was performed in a Corbett Rotor-gene 6000 thermocycler (Corbett Lifesciences, Sydney). mRNA expression was assessed by the $\Delta \Delta \mathrm{Ct}$ method.

Statistical analysis

Data was statistically analyzed using Prism 4.03 software. $T$ test was used to compare results between experimental groups.

\section{Results}

In a first step, the concentration of tryptophan and kynurenine was measured in the iLN taken from naïve WT and healthy Ido1KO mice. However, it was found that neither the concentration of tryptophan nor the concentration of kynurenine was affected in iLN by the deletion of Idol, Table 1. The lack of changes in the concentration of tryptophan and kynurenine in naïve iLN could be explained by the fact that loss of IDO1 function may be compensated by the increased expression of IDO2. Moreover, in contrast to what could be expected, it was found that mRNA expression for Ido2 was significantly decreased $(p<0.05)$ in iLN taken from naïve Ido1KO mice (Fig. 2a).

In a next step it was of interest to test if CIA could impact the concentration of tryptophan and kynurenine in iLN taken from WT animals with CIA and diseased Ido1KO mice. Interestingly, the mean concentration of tryptophan was found to be significantly $(p<0.05)$ increased in iLN taken from Ido1KO mice with CIA in comparison with tissues harvested from WT arthritic animals, Table 1. Moreover, the concentration of kynurenine in iLN was not affected by CIA, Table 1 . Next, mRNA expression for Ido2 was assessed in iLN from arthritic mice. However, mRNA expression for Ido2 was also significantly $(p<0.001)$ reduced in the iLN taken from Ido1KO mice with CIA in comparison with iLN taken from WT diseased mice (Fig. 2b).

It was also interesting to check if deletion of $I d o l$ could impact the concentration of kynurenine in serum of arthritic mice. However, it was found that in Ido1KO mice with CIA the concentration of kynurenine was significantly $(p<0.001)$ decreased in comparison with WT mice with CIA. In sera of arthritic WT mice the mean concentration of kynurenine was $1.83 \pm 0.24 \mu \mathrm{M}$. In contrast, in sera of Ido1KO mice with CIA the mean concentration of kynurenine dropped to $0.61 \pm 0.11 \mu \mathrm{M}$.

In addition, it was also of interest to test if an inactivation of IDO1 and decreased mRNA expression for Ido2 in iLN from Ido1KO mice with CIA could be compensated by the changes in the mRNA expression for downstream genes (Afm, Kmo, Kynu, and Haao) on the kynurenine pathway. However, no such effect was observed in iLN from Ido1KO mice with arthritis in comparison with WT mice with CIA (supplementary figure 1). Similarly, in iLN taken from neither naïve nor from the diseased Ido1KO animals, the concentration of kynurenine catabolites, AA and 3-HAA, was significantly changed (supplementary table 2).

\section{Discussion}

The aim of this work was to investigate the impact of Idol deletion on the concentration of tryptophan and its biologically active catabolites: kynurenine, AA, 
Table 1 Concentration of tryptophan and kynurenine in iLN from WT and Ido1KO mice

\begin{tabular}{llccc}
\hline Compound & $\begin{array}{l}\text { Naive WT mice } \\
\text { (nmol/g wet tissue) }\end{array}$ & $\begin{array}{l}\text { Naive Ido1KO mice } \\
(\mathrm{nmol} / \mathrm{g} \text { wet tissue) }\end{array}$ & $\begin{array}{l}\text { Arthritic WT mice } \\
\text { (nmol/g wet tissue) }\end{array}$ & $\begin{array}{l}\text { Arthritic Ido1KO mice } \\
\text { (nmol/g wet tissue) }\end{array}$ \\
\hline Tryptophan & $5.11 \pm 0.85$ & $6.07 \pm 3.8$ & $5.12 \pm 0.4$ & $8.68 \pm 2.4 *$ \\
Kynurnine & $731 \pm 250.7$ & $618.7 \pm 231$ & $998 \pm 709$ & $589.7 \pm 198.1$ \\
\hline
\end{tabular}

iLN were isolated from naive and mice with CIA of C57BL/6J strain and Ido1KO mice, $\mathrm{n}=5$ for each strain and conditions. The concentration of tryptophan was measured with HPLC method, whereas the concentration of kynurenine was measured with a colorimetric assay. Results were assessed using $t$ test

$* p<0.05$

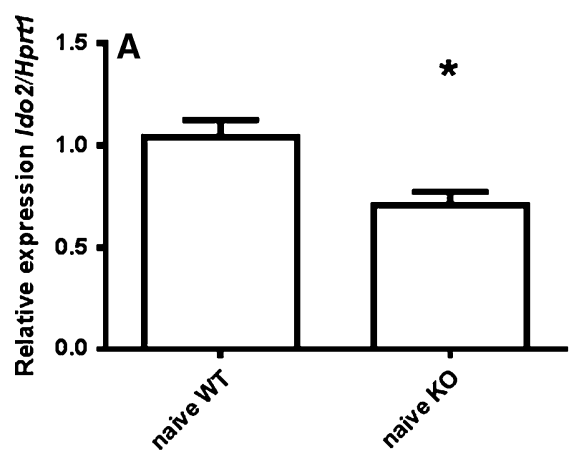

Fig. 2 Decreased mRNA expression for Ido2 iLN from naïve and arthritic Ido1KO mice in comparison with WT controls. CIA was induced in C57BL/6J mice $(n=5)$ and Ido1KO animals $(n=5)$. Ten days after onset of the disease have been spotted animals were sacrificed and iLN isolated. mRNA expression for Ido2 was assessed using qRT-PCR and results

and 3-HAA in naïve iLN isolated from WT and Ido1KO mice as well as those taken from animals with CIA. In addition, the concentration of kynurenine was measured in sera of Ido1KO mice with CIA and WT arthritic animals. Metabolic data was also supported by the results showing mRNA expression for other genes on the kynurenine pathway in iLN upon Idol deletion.

Interestingly, it was found that in naïve iLN taken from Ido1KO mice the concentration of tryptophan was not affected by the deletion of Idol. In contrast, tryptophan was accumulated in iLN of Ido1KO mice upon immune challenge driven by CIA. Increased accumulation of tryptophan in iLN from Ido1KO mice during CIA may also emphasize a functional importance of IDO1 in the regulation of tryptophan concentration in the local tissue environment (Lob and Konigsrainer 2009; Lob et al. 2009). It is known that upon specific conditions (e.g. acidosis) tryptophan can be displaced from albumin (Cunningham et al. 1975).

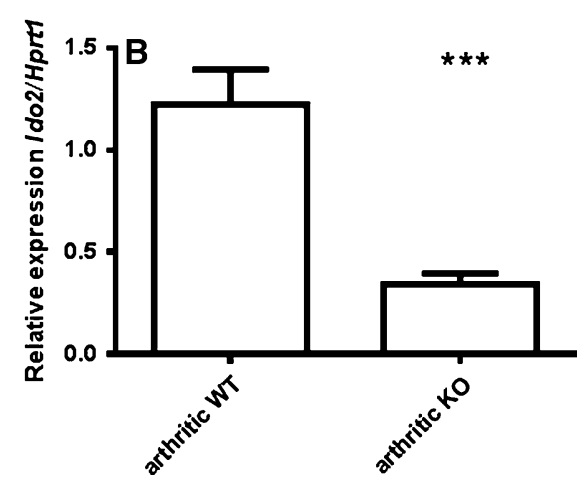

were compared with naïve IDO1KO mice $(\mathrm{n}=5)$ and naïve WT C57BL/6J animals $(\mathrm{n}=5)$. Data was analyzed using $t$ test. a Significantly decreased Ido2 mRNA expression in iLN taken from naïve Ido1 KO mice b significantly decreased Ido2 mRNA expression in iLN taken from Ido1KO mice with CIA $* p<0.05 ; * * * p<0.001$

This process may impact the metabolic flux through the kynurenine pathway (Smith and Pogson 1980). Hence, increased concentration of tryptophan upon deletion of Idol suggests that in deed IDO1 can regulate concentration of tryptophan in the local tissue environment. In addition, in the previous papers, I have shown that the full anti inflammatory potential of the kynurenine pathway is likely to be achieved upon coincidence between decreased concentration of tryptophan and accumulation of kynurenines in iLN (Kolodziej 2012) but not in the serum (Kolodziej 2013).

IDO2 is a relatively recently discovered enzyme which can also mediate oxidative catabolism of tryptophan (Metz et al. 2007). However, physiological importance of this enzyme remains elusive. Thus, Ido1KO mice could be a useful tool in the dissection of IDO2 role in the regulation of tryptophan catabolism. Moreover, here, it has been found that mRNA expression for Ido2 was decreased in the iLN taken from naïve Ido1KO mice as well as those with CIA. 
Hence, Ido1KO mice may not be particularly suitable for investigations of a physiological role of IDO2.

Here, it has been also found that deletion of Idol and significantly reduced Ido2 mRNA expression did not result in the abnormally changed concentration of kynurenine and its anti inflammatory catabolites: AA and 3-HAA in the naïve iLN. Similarly, mRNA expression for downstream genes on the kynurenine pathway: Afm, Kmo, Куnu, and Haao were found to be not changed in iLN taken from Ido1KO mice with CIA. However, it has to be also acknowledged that the kynurenine pathway also consists of metabolic branches on which biologically active metabolites have been produced (Amori et al. 2009). It has been shown that kynurenic acid, derived from kynurenine, influenced leucocytes (Barth et al. 2009). Moreover, due to technical and financial limitations in this project, it was not possible to investigate metabolic flux through branches on the kynurenine pathway.

The concentration of kynurenine was discovered to be significantly decreased in sera of Ido1KO mice with CIA. This observation may be explained by two distinct but not entirely exclusive possibilities. It may be that upon immune challenge deletion of IdoI is sufficient enough to cause a decreased concentration of kynurenine in serum but not in the iLN. Alternatively, it has been shown kynurenine can be actively transported into the T cells via the CD98 transporter (del Amo et al. 2008). Hence, under reduced rate of kynurenine anabolism, this process could account for decreased concentration of kynurenine in serum (Kolodziej et al. 2011). Moreover, this theory needs to be experimentally tested yet.

Nonetheless, taken together, in this study it was shown deletion of IdoI alongside with decreased Ido2 mRNA expression resulted in the accumulation of tryptophan in the iLN and reduced concentration of kynurenine in sera of transgenic animals with CIA. These results support the importance of IDO enzymes in the regulation of tryptophan concentration in the local tissue environment and immune system.

Acknowledgments The author would like to thank KIR Trustees and Arthritis Research Campaign UK for their financial support. Dr Robert Visse from the Kennedy Institute of Rheumatology has provided an excellent technical support with HPLC analyses, whereas Dr Richard Williams and Ewa Paleolog thought me CIA model.

Conflict of interest The author declare no competing of interests.
Open Access This article is distributed under the terms of the Creative Commons Attribution License which permits any use, distribution, and reproduction in any medium, provided the original author(s) and the source are credited.

\section{References}

Amori L et al (2009) On the relationship between the two branches of the kynurenine pathway in the rat brain in vivo. J Neurochem 109(2):316-325

Baban B et al (2004) Indoleamine 2, 3-dioxygenase expression is restricted to fetal trophoblast giant cells during murine gestation and is maternal genome specific. J Reprod Immunol 61(2):67-77

Baban B et al (2009) IDO activates regulatory T cells and blocks their conversion into Th17-like T cells. J Immunol 183(4): 2475-2483

Ball HJ et al (2007) Characterization of an indoleamine 2, 3 -dioxygenase-like protein found in humans and mice. Gene 396(1):203-213

Barth MC et al (2009) Kynurenic acid triggers firm arrest of leukocytes to vascular endothelium under flow conditions. J Biol Chem 284(29):19189-19195

Criado $\mathrm{G}$ et al (2009) Indoleamine 2, 3 dioxygenase-mediated tryptophan catabolism regulates accumulation of Th1/Th17 cells in the joint in collagen-induced arthritis. Arthritis Rheum 60(5):1342-1351

Cunningham VJ, Hay L, Stoner HB (1975) The binding of Ltryptophan to serum albumins in the presence of nonesterified fatty acids. Biochem J 146(3):653-658

del Amo EM, Urtti A, Yliperttula M (2008) Pharmacokinetic role of L-type amino acid transporters LAT1 and LAT2. Eur J Pharm Sci 35(3):161-174

Desvignes L, Ernst JD (2009) Interferon-gamma-responsive nonhematopoietic cells regulate the immune response to Mycobacterium tuberculosis. Immunity 31(6):974-985

Fallarino F et al (2002) T cell apoptosis by tryptophan catabolism. Cell Death Differ 9(10):1069-1077

Fallarino F et al (2006) The combined effects of tryptophan starvation and tryptophan catabolites down-regulate $\mathrm{T}$ cell receptor zeta-chain and induce a regulatory phenotype in naive T cells. J Immunol 176(11):6752-6761

Frumento $G$ et al (2002) Tryptophan-derived catabolites are responsible for inhibition of $\mathrm{T}$ and natural killer cell proliferation induced by indoleamine 2, 3-dioxygenase. J Exp Med 196(4):459-468

Hara $\mathrm{T}$ et al (2008) Diazotization of kynurenine by acidified nitrite secreted from indoleamine 2, 3-dioxygenaseexpressing myeloid dendritic cells. J Immunol Methods 332(1-2):162-169

Hayaishi O (1976) Properties and function of indoleamine 2, 3-dioxygenase. J Biochem 79(4):13P-21P

Inglis JJ et al (2007) Collagen-induced arthritis in C57BL/6 mice is associated with a robust and sustained T-cell response to type II collagen. Arthritis Res Ther 9(5):R113

Kolodziej L (2012) An exploratory study of the interplay between decreased concentration of tryptophan, 
accumulation of kynurenines, and inflammatory arthritis. IUBMB Life 64(12):983-987

Kolodziej L (2013) Systemic metabolism of tryptophan and its catabolites, kynurenine and 3-HAA, in mice with inflammatory arthritis. Gene 512(1):23-27

Kolodziej LR, Paleolog EM, Williams RO (2011) Kynurenine metabolism in health and disease. Amino Acids 41(5): 1173-1183

Lob S, Konigsrainer A (2009) Role of IDO in organ transplantation: promises and difficulties. Int Rev Immunol 28(3-4): 185-206

Lob S et al (2009) Inhibitors of indoleamine-2, 3-dioxygenase for cancer therapy: can we see the wood for the trees? Nat Rev Cancer 9(6):445-452

Metz R et al (2007) Novel tryptophan catabolic enzyme IDO2 is the preferred biochemical target of the antitumor indoleamine 2, 3-dioxygenase inhibitory compound D-1-methyLtryptophan. Cancer Res 67(15):7082-7087

Moffett JR, Namboodiri MA (2003) Tryptophan and the immune response. Immunol Cell Biol 81(4):247-265

Munn DH et al (1999) Inhibition of $\mathrm{T}$ cell proliferation by macrophage tryptophan catabolism. J Exp Med 189(9): $1363-1372$

Park MJ et al (2011) Transforming growth factor beta-transduced mesenchymal stem cells ameliorate experimental autoimmune arthritis through reciprocal regulation of Treg/Th17 cells and osteoclastogenesis. Arthritis Rheum 63(6):1668-1680
Russo S et al (2003) Tryptophan as a link between psychopathology and somatic states. Psychosom Med 65(4):665-671

Sato K et al (2006) Th17 functions as an osteoclastogenic helper $\mathrm{T}$ cell subset that links $\mathrm{T}$ cell activation and bone destruction. J Exp Med 203(12):2673-2682

Sharma MD et al (2009) Indoleamine 2, 3-dioxygenase controls conversion of Foxp3 + Tregs to TH17-like cells in tumordraining lymph nodes. Blood 113(24):6102-6111

Smith SA, Pogson CI (1980) The metabolism of L-tryptophan by isolated rat liver cells. Effect of albumin binding and amino acid competition on oxidatin of tryptophan by tryptophan 2, 3-dioxygenase. Biochem J 186(3):977-986

Smolen JS, Aletaha D (2009) Developments in the clinical understanding of rheumatoid arthritis. Arthritis Res Ther 11(1):204

Terness P et al (2002) Inhibition of allogeneic T cell proliferation by indoleamine 2, 3-dioxygenase-expressing dendritic cells: mediation of suppression by tryptophan metabolites. J Exp Med 196(4):447-457

Ueda T et al (1978) The metabolism of [carboxyl-14C]anthranilic acid. I. The incorporation of radioactivity into NAD+ and NADP+. J Biochem 84(3):687-696

Weaver CT et al (2006) Th17: an effector CD4 T cell lineage with regulatory $\mathrm{T}$ cell ties. Immunity 24(6):677-688

Zaiss MM et al (2007) Treg cells suppress osteoclast formation: a new link between the immune system and bone. Arthritis Rheum 56(12):4104-4112 